\title{
Enhancing the seismic histogram equalization of multi-fusion for infrared image of concealed weapon detection
}

\author{
Nashwan Jasim Hussein ${ }^{a}$, Fei Hu, Feng He and Ayoob Azeez Ayoob \\ School of Electronic Information and Communication, National Key Laboratory of Science and Technology \\ Huazhong University of Science and Technology, Wuhan 430074, China
}

\begin{abstract}
The objective of concealed weapon detection is to detect and recognize individuals or groups of terrorists in public areas, such as airports,trains, railways, and even malls. This work investigates how the lives of people in crowded areas can be protected with respect to humanitarian privacy. Seismic histogram equalization is used to enhance captured images and reveal any weapons placed underneath clothes. Infrared images, which have minimum ratios of mistake, are utilized to guarantee safety.In this paper novel methodology proposed for robust detection weapons hidden in clothes by using color image and its infrared image as input and then applying process of image fusion using discrete wavelet transform (DWT) and segmentation using thresholding method. The infrared (IR) image is used as it helps to get visual information about hidden weapons. The experimental results presented are prepared by using real time RBG and IR images those are captured by infrared sensors. The experimental result of this work clearly the solution of how to detect of concealed weapon underneath person cloths with minimum false and high level of privacy this work relies on infrared images that contain all of the information about weapons carried underneath. The experiment results are based on the enhanced seismic histogram of infrared images.
\end{abstract}

Keywords: image enhancement; image seismic; weapon detection; pinpoint area.

\section{Introduction}

Concealed weapon detection has gained considerable attention in scientific fields[1]. Many military and civil institutions have focused on this topic after terrorist organizations have attacked countries, leaving its citizens and law-enforcing agencies shell-shocked and causing severe damages to infrastructures and the environment. Since then, countries that have experienced attacks have attempted to develop effective detection devices that can implement proactive terrorist operations. Image processing has substantially contributed to the development of sensor devices because of this technique's robustness, effectiveness, modest illumination, and easy implementation [18]. The main object of this study is the infrared image[2]. The radiation emitted from hardware infrared devices collides with the body and then returns to the emitted [3] resource. The degree of IR image resolution depends on the radiation reflected from the body exposed to IR senescing. The mental image is used for channel extraction, image fusion, and pre-processing. However, the color image is the one that provides the visual perception[4] about the desired object[19-20]. This study focuses on the

${ }^{a}$ Correspondence author : wuyumin005@163.com 
enhancement of IR images because such images are mainly used for day and night monitoring of people and vehicles at checkpoints. However, such monitoring is hindered by observational zero vision because of the inability of IR radiation to describe and specify the details of groups or individuals and their movements without disturbing human privacy [5]. The main process in this work is the image enhancement technique, which is a clarifying process of visual perception. It can define enhancement as group of techniques containing image processing enhancement and image-enhancing processes that do not change the natural visibility[6]. The underlying processes of the enhancement process have two types: direct and indirect. Direct enhancement involves the manipulation of image pixels and enhancing it, whereas indirect enhancement involves seeking out the coefficient and inverse or modifying it. The concealed weapon detection technique relies on the robustness of the hardware [7]. The current work applied traditional image processing techniques of resizing, combining, converting and image fusion to deliver the images captured to the intended imageenhancing process.

\section{Literature review}

Many studies have explored concealed weapon detection. Image enhancement has become one of the main methods for solving the image resolution problem and supporting images captured from far distances or cases of haze weather. Moreover, image enhancing techniques have been extensively used in the medical domain before being applied to detect objects underneath. Jun Yin, Xiaolin Tian, Zesheng Tang, Yankui Sun et al. attempted to use the fast algorithm to enhance of images captured for tumors [8]. Many other studies have explored this method for human treatment. After the huge shift to maintain security and ensure public safety, image processing techniques played a key role in image manipulation via contrast enhancement. Histogram equalization is one of the main methods for more efficiently capturing images in different ways and creating new images for detection or understanding the hidden details of image targets. Manpreet Kaur et al. focused on using histogram equalization Krishna et al[9]. Also used histogram equalization to manipulate the brightness of images, that is, controlling the changing of light after histogram equalization is performed [10]. Many others researchers have attempted to overcome the changes in brightness between the input and output images by more modest techniques, such as dualistic histogram equalization, peak signal-to-noise ratio, and bi-histogram equalization. Omprakash Patel et al. attempted to change the disadvantages of the image brightness caused by histogram equalization[11].

\section{Methodology}

Concealed weapon detection is critical topic due of the effectiveness to capture images by senescing devices may not be enough to clearly reveal weapons kept. It supports the senescing device with builtin software to enhance the output result and utilize image processing properties. The important idea in this domain is finding a way to maintain the required details after an image is processed, because the enhancement causes changes to the details. In this case, the undesired information of the image will be cleared, and the required glance will disappear [12]. Early enhancing methods changed the brightness of images without analysing which areas require increased or decreased brightness. Such a case results in more shine, rendering the enhanced image un-useful. Many studies have developed different sophisticated methods can give the desired result with modest changes in brightness or details. Recent methods can be used to enhance images with different environments and make the senescing device more comfortable by supplying it the required software. The current work offers two main enhancement equalization methods, namely, global enhancing and local enhancing, for creating the image after fusion. The image is then enhanced without changing the important details. 


\subsection{Proposed method}

In literature we have studied number of weapon detection techniques with different limitations such as lack of accuracy, less efficiency, more computational overhead etc. Therefore to alleviate the accuracy, efficiency, computational overhead related problems, we are contributing the novel framework of automatic weapon detection based on methodology of image fusion, image morphology and other image processing techniques. In this section detailed process and methods proposed for efficient weapon detection are discussed. Basically in first step is to get image acquisition where system taking two images RGB image and IR image. After that pre-processing operation performed on both images, then fusion is done, finally segmentation and edge detection is performed to display the result of weapon detection. The algorithm and its flowchart showing the major contribution of this research work mentioned below. The uniqueness of this approach is that, it is combination of methods like histogram equalization; value channel of HSV of color space, performing the DWT based fusion of histogram equalization result of IR image and V channel of color image. To improve the contrast again fused image is enhanced. To get weapon detection part more clearly thresholding and contrast enhancement perform. The proposed method of concealed weapon detection fall in ten steps, the fourth steps of it preparing the images to make it ready to the fusion step that (fusion step) play the key role for other fifth proposed steps. The resolution of both infrared image and color image is used is of size $256 * 256$.

Algorithm 1: Efficient Weapon Detection

Input 1: Color Image

Input 2: Infrared Image

Output: Enhanced Weapon Detection Results

Step 1: Resize the captured images (IR and visual images) as shown in figure 1.

Step 2: HE for IR image (to increase brightness).

Step 3: Extract V channel from color image.

Step 4: Conduct fusion between V channel and IRHE.

Step 5: Enhance fusion images.

Step 6: Use threshold algorithm to extract the contour of HE of enhanced fusion images.

Step 7: Combine contour with enhanced fusion images.

Step 8: Enhance image contrast.

Step 9: Extract image from local histogram enhancement (LHE).

Step 10: Final results of enhanced images.

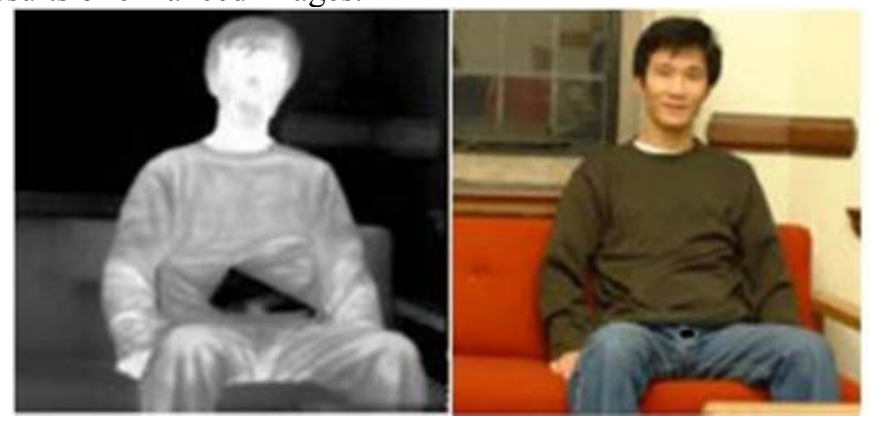

Figure 1. It is showing the dataset image of concealed weapon detection left to right, infrared image or informative image that have all information need it after manipulate with fusion and enhance steps. 


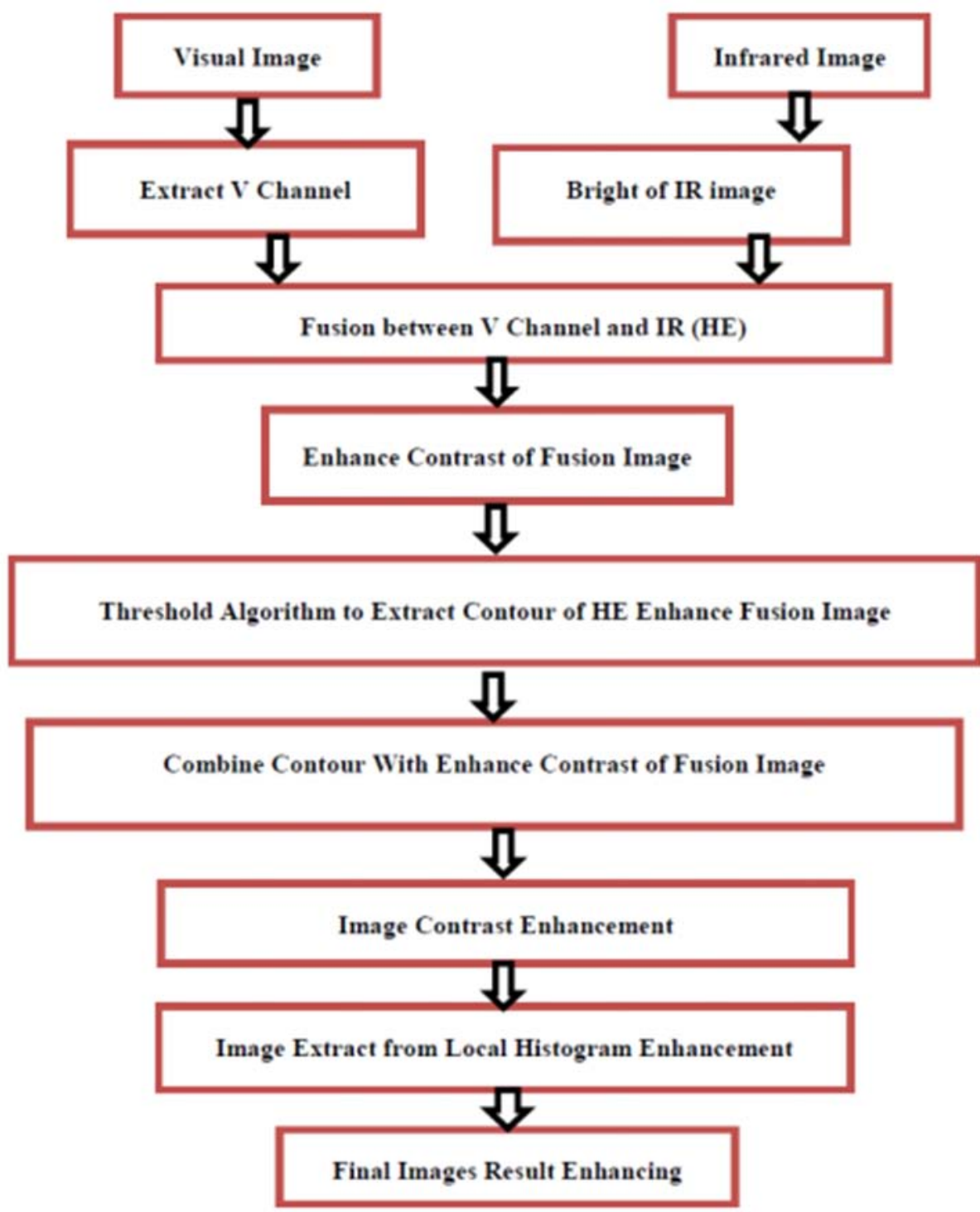

Figure 2.

It is showing the flowchart of proposed system. Proposed method for images enhancement explain the step by step of capturing and doing the process of fusion and multi enhancement process in different level to get high precise result of detection. This figure is explaining inputs to output process for weapon detection. Figure 3 below is showing the cycle of image enhancing with sub-step enhancing to explain to extract the final images enhancing using global and local enhancement. LEE: Local equalization enhancement, GEE: Global equalization enhancement. The detailed process of weapon detection approach is showing in figure 3 which is starting from image browsing, then channel extraction, then histogram equalization, then fusion and image segmentation with results. The final output is showing the weapon detection on input color image. It shows the two different results for weapon detection with their histograms. Left result histogram is having less contrast whereas right result histogram having improved contrast. 


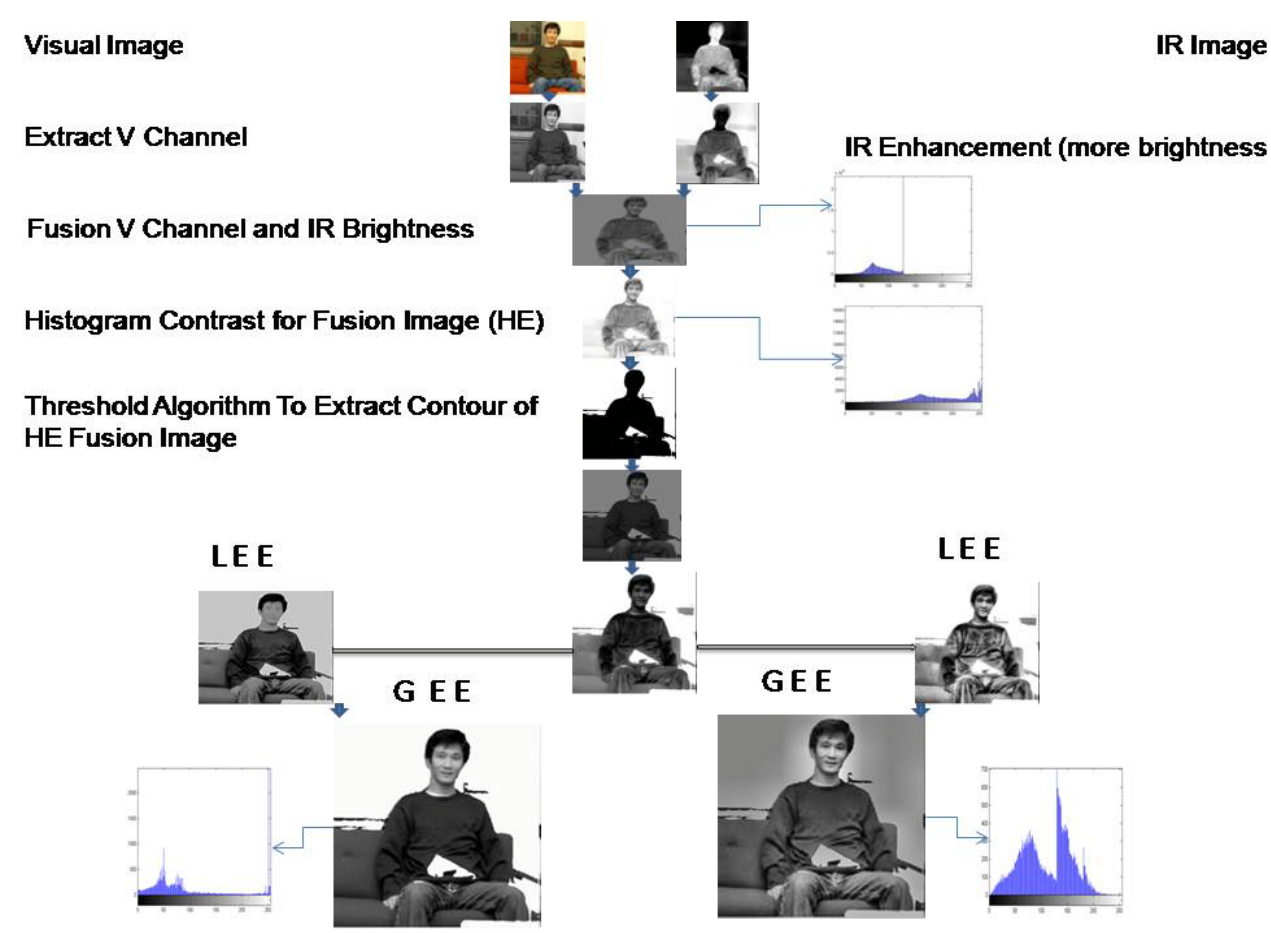

Figure 3. Practical workflow of proposed weapon detection

\subsection{Seismic enhancement histogram local and global enhancing}

In this paper, dynamic range seismic image enhancement is used to process a traditional image (resizing, format conversion, fusion processing) before the enhancement process is conducted. Local and global enhancement plays key roles in obtaining results with more details about the hidden objectives. The difference between seismic histogram equalization and histogram equalization is that seismic histogram equalization supporting the adaptive and dynamic contrast enhancement based on input image which is not the case with histogram equalization function.

\subsection{Global enhancement equalization}

The idea global enhancement equalization (GEE) is to prepare the images for local enhancement equalization (LEE). Figure (4) shows the changing of the seismic histogram. In GEE, the input divides the image into two parts. These two parts are derived based on min-max threshold values of contrast enhancement. Then, dynamic stretching is performed on the input image, and the changes in the output images will be clear for the mental vision with different gradients of brightness. The equation of GEE is shown in Eq. 1. Both images provide clear views that a change happened and that the dynamic density of the images had been changed. The histogram of the left image jumped because the sample of histogram grew modestly from 0 to more than 165[14-15]. The sample makes a hard jump in area of the hidden object (the area of the concealed weapon) because the reflection of metal for infrared radiation becomes more active in this area[16-17]. The same scenario can be observed in the right image. 


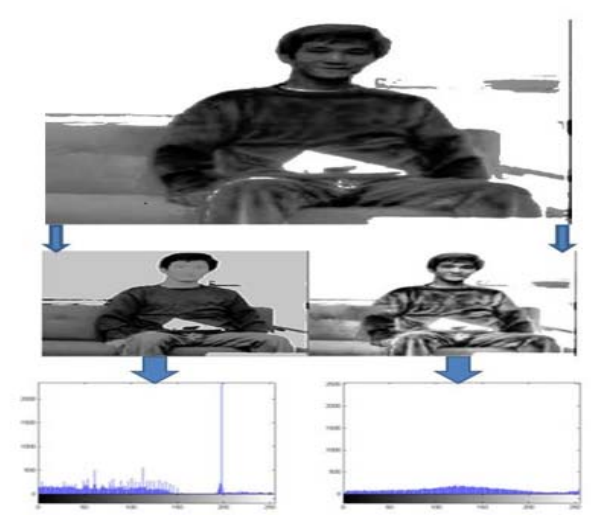

Figure 4. It is showing the results of histogram equalization in which both images histograms presented in order to show the difference between them.

$$
\mathrm{T}(\mathrm{g})=\operatorname{gmin}+(\text { gmaximum }- \text { gminimum }) * \frac{\sum_{\mathrm{x}}^{\mathrm{g}} \operatorname{gminh}(\mathrm{x})}{\sum_{\mathrm{x}}^{\mathrm{gmax}}=\operatorname{gmin} \mathrm{h}(\mathrm{x})}
$$

1- $\quad g$ is the intensity of the image.

2- $\quad g$ min and g max denote the minimum and maximum values for the histogram of the image.

3- $\quad \mathrm{T}(\mathrm{g})$ is the unit of transformation.

4- $\quad x$ is the image

5- $\mathrm{h}(\mathrm{x})$ is its histogram

The max and min value reflect the image intensity from (0: modest values, 255: $\max )$.For improvement in results, we have designed and developed both global enhancing and local enhancing methods.

\section{Discussion and results}

Both results taken from GEE proceed to second step of enhancement (LEE) to combine the enhancing methods together (GEE and LEE) and stretch the dynamic range and control the seismic histogram of the image.

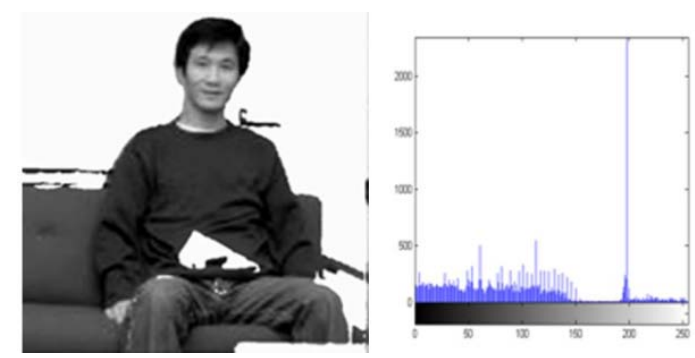

\section{Figure 5.}

It is showing the result of weapon detection at first attempt based on histogram equalization, segmentation and fusion. The global images obtained from the processing of GEE enhancement equalization with general lighting can modify and enhance separately to obtain different results to the equation of local and global enhancement. The below equation 2 is used for deriving the image enhancement after applying global image enhancement.

$$
\mathrm{Fv}(\mathrm{x}, \mathrm{y})=255 *[\mathrm{Io}(\mathrm{x}, \mathrm{y})-\operatorname{minimum}] /(\operatorname{maximum}-\operatorname{minimum})
$$


Where $\mathrm{Fv}(\mathrm{x}, \mathrm{y})$ is the intensity pixel of the output image after the dynamic range is stretched. Io (x, $y)$ is the intensity pixel of the input image after global enhancement equalization.Minimum and maximum refers to the max and min values for image pixel intensity .
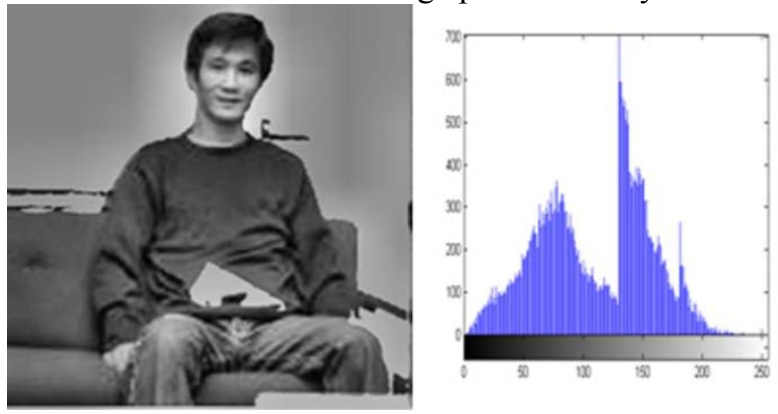

\section{Figure 6.}

The results obtained by LEE showing in figure 6 above, that is, the changing of seismic histogram, clearly shows that the important area (area of hidden weapon) has a high degree of changing with respect to the other areas in an image. The sample of the histogram grew fast and suddenly in place of the hidden object to give the desired information about the metal or carried weapon. The final results give a clear perspective of the object and make the target clearer.

\section{Conclusion}

Weapon detection in public areas is becoming most widely focused research area since from last decade for researchers with goal of automatic detection of hidden weapons in order to prevent the harmful actions and provide security to individual or group of individuals or assets. In this paper, novel method is proposed for efficient framework hidden weapon detection using RGB and its IR image as input. The methods used are HSV color space, image fusion using DWT, segmentation using thresholding method, canny edge detector for estimating the final result of weapon detection on color image. The main dataset in our work is the color image, which only has the visual. The IR image serves as the main object of our work, and the success of the fusion and enhancement process depend on the IR image. The enhancement processes were applied successfully by using image processing operation, and the results were satisfactory with an acceptable percentage. This paper introduced two different approaches and its results are also shown. For future work, we suggest to deploy proposed approach under real time settings for detection weapons automatically.

\section{References}

1. $\mathrm{Yu}, \mathrm{W} ., \mathrm{X}$. Chen, and L. Wu, Segmentation of Concealed Objects in Passive Millimeter-Wave Images Based on the Gaussian Mixture Model. Journal of Infrared, Millimeter, and Terahertz Waves. 36(4): p. 400-421, (2015)

2. Achanta, A.; McKenna, M.; Heyman, J.; Rudd, K.; Hinders, M.; Costianes, "Non-linearAcoustic Concealed Weapons Detections" 34th Applied Imagery and Pattern Recognition Workshop (AIPR05), (2005).

3. Baum, C. E.; "on the singularity expansion method for the solution of electromagnetic interaction problem" Air Force Weapons Lab. Interaction Notes, Note 88, Dec. (1971).

4. Dr. Mohamed Mansoor Roomi and R. Rajashankari, "Detection of concealed weapons inX-ray Images using Fuzzy K-NN", International Journal of Computer Science, Engineeringand Information Technology (IJCSEIT), Vol. 2, No.2, April 2012. 
5. Xiaodong Zhuge and Alexander G. Yarovoy, a Sparse Aperture MIMO-SAR-BasedUWB Imaging System for Concealed Weapon Detection. IEEE Transaction on geoscienceand remote sensing vol. 49, no. 1, January 2011.

6. Parande, M. and S. Soma, Concealed Weapon Detection in a Human Body by Infrared Imaging.

7. Kotkar, V.A. and S.S. Gharde, Review of various image contrast enhancement techniques. International Journal of Innovative Research in Science, Engineering and Technology. 2(7): p. 2786-2793, (2013)

8. Cho, S.-Y. and N.-P. Tin. Using infrared imaging technology for concealed weapons detection and visualization. in TENCON 2010-2010 IEEE Region 10 Conference. IEEE. (2010)

9. Yin, J., et al. A histogram-based fast enhancement algorithm for CT head images. in Biomedical and Pharmaceutical Engineering, 2006. ICBPE 2006. International Conference on. 2006. IEEE.

10. Kaur, M., J. Kaur, and J. Kaur, Survey of contrast enhancement techniques based on histogram equalization. IJACSA) International Journal of Advanced Computer Science and Applications. 2(7), (2011)

11. Krishna, A.S., G.S. Rao, and M. Sravya, CONTRAST ENHANCEMENT TECHNIQUES USING HISTOGRAM EQUALIZATION METHODS ON COLOR IMAGES WITH POOR LIGHTNING. International journal of computer science, engineering and applications. 3(4): p. 15, (2013)

12. Patel, O., Y.P. Maravi, and S. Sharma, A comparative study of histogram equalization based image enhancement techniques for brightness preservation and contrast enhancement. arXiv preprint arXiv:1311.4033, (2013).

13. Jen, T.-C. and S.-J. Wang. Generalized histogram equalization based on local characteristics. in Image Processing, 2006 IEEE International Conference on. IEEE. (2006)

14. Xue, Z. and R.S. Blum. Concealed weapon detection using color image fusion. in Proceedings of the 6th International Conference on Information Fusion. (2003).

15. Boschetti, A., et al. High dynamic range image tone mapping based on local histogram equalization. in Multimedia and Expo (ICME), 2010 IEEE International Conference on. IEEE. (2010)

16. Tian, Y., Q. Wan, and F. Wu. Local histogram equalization based on the minimum brightness error. in null. IEEE. (2007)

17. Nelson, C. V.; "Metal Detection and Classification Technologies" Johns Hopkins APL technical Digest, Vol. 24, Number 1, (2004).

18. Imran Memon, Ling Chen, Abdul Majid,"Travel Recommendation Using Geo-tagged Photos in Social Media for Tourist” Wireless Personal Communications (2015).

19. Muhammad Hammad Memon, "Content Based Image Retrieval Based On Geo-Location Driven Image Tagging On The Social Web", International Conference on Wavelet Active Media Technology and Information Processing 2014(ICCWAMTIP 2014) on December 19-21, 2014. Pages: 280 - 283, DOI: 10.1109/ICCWAMTIP.7073408.(2014)

20. MH Memon, "Unsupervised Feature Approach For Content Based Image Retrieval Using Principal Component Analysis", International Conference on Wavelet Active Media Technology and Information Processing 2014(ICCWAMTIP 2014) on December 19-21, 2014. ICCWAMTIP 2014, p 271-275, DOI: 10.1109/ICCWAMTIP.7073406.(2014) 